\title{
La filmación de clase en contexto de colegialidad: estrategia que favorece los procesos reflexivos respecto de la práctica pedagógica
}

\section{The Class Filming in Collegiate Context: A Strategy that Favors The Reflective Processes on the Pedagogical Practice}

Jessica Bórquez-Mella*

Universidad Católica de Temuco, Chile.

ORCID: https://orcid.org/0000-0001-7954-5983

Sandra Garrido-Osses

Universidad Católica de Temuco, Chile.

ORCID: https://orcid.org/0000-0003-1419-9287

Luzmila Flores-Gajardo

Universidad Católica de Temuco, Chile.

ORCID: https://orcid.org/0000-0003-1296-4635

Recibido 02-01-20 Revisado 15-02-20 Aprobado 30-03-20 En línea 07-04-20

*Correspondencia

Email: jeborquez@uct.cl
Citar como:

Bórquez-Mella, J., Garrido-Osses, S., \& Flores-Gajardo, L. (2020). La filmación de clase en contexto de colegialidad: estrategia que favorece los procesos reflexivos respecto de la práctica pedagógica. Propósitos y Representaciones, 8(SPE1), e500. doi: http://dx.doi.org/10.20511/pyr2020.v8nSPE1.500 


\section{Resumen}

En las últimas décadas, la reflexión respecto de las prácticas profesionales docentes ha sido considerada como un medio de desarrollo importante entre los formadores de profesores. Sin embargo, investigaciones chilenas dan cuenta que las universidades de este país no han logrado que los estudiantes de pedagogía desarrollen sistemáticamente reflexiones en relación a su quehacer docente. Esta situación no se aleja de la realidad que viven los estudiantes de la carrera de Pedagogía Media en Ciencias Naturales y Biología de la Universidad Católica de Temuco. Estos logran solo en algunos casos, y de forma intuitiva, reflexiones para la mejora de su mejorar desempeño. El propósito de esta investigación es fortalecer los niveles de reflexión de estudiantes de pedagogía. En este contexto, la investigación es cualitativa con diseño de estudio de caso único y evidenció la relevancia de la utilización de filmaciones de clase como estrategia que favorece procesos reflexivos sobre la práctica pedagógica en contextos de colegialidad. Se concluyó que los participantes valoran la estrategia de filmación de clase dado que favorece la autoevaluación del desempeño. Asimismo, se observó una movilización de los niveles de reflexión de la práctica pedagógica desde el nivel descriptivo al crítico; sin embargo, cabe destacar que se genera la movilización señalada dependiendo del tiempo que destinen los participantes al proceso reflexivo.

Palabras clave: Filmación de clase; Formación de profesores; Reflexión práctica docente.

\section{Summary}

In recent decades, the reflection on the teaching practices has been considered as an important means among the teacher trainers. Nevertheless, chilean research note that the universities of this country have not achieved that students of Teaching Programs may develop reflections on their pedagogical procedures systematically. This situation is no far from the reality of students of Pedagogía Media en Ciencias Naturales y Biología from Universidad Católica de Temuco. They achieve only in some cases, and intuitively, reflections that may help them improve their performance. The purpose of this research is to strengthen the levels of students' reflection. In this context, the investigation is a qualitative one with a unique case study design. It points to the relevance of the use of class filming as a strategy which favors reflective processes on the pedagogical practice in collegiate contexts. It concludes that the participants appreciate the strategy of class filming, given that it favors the self assessment of their performance. Likewise, a mobility of the levels of reflection from the descriptive to the critic one, is shown. Nevertheless, it is important to highlight that the mobility depends on the time the participants spend on the reflective process.

Keywords: Class Filming; Teacher Training, Reflection on Teaching Practice.

\section{Introducción}

La práctica profesional docente, es entendida como una instancia en la que estudiantes de pedagogía se integran al mundo escolar y constituye un punto decisivo en la formación inicial de profesores. Durante el desarrollo de esta práctica donde los estudiantes se desenvuelven por primera vez en su nuevo rol como docentes y, por to-tanto, es un espacio que permite vivenciar experiencias profesionales nuevas y se perfila como en el punto de partida desde el que se construyen reflexiones sobre el actuar docente. Así, Correa (2011) reconoce a la práctica profesional como un espacio que contribuye tanto a la profesionalización como al desarrollo profesional de los estudiantes de pedagogía. Para Montecinos (2011) releva la práctica docente como un aspecto clave en la formación inicial de profesores, puesto que en ella los estudiantes de pedagogía deben movilizar saberes, actitudes, valores y funciones que han recibido en su vida universitaria. Otra evidencia de la importancia del proceso en cuestión es el hecho de que la mayoría de los docentes y estudiantes de pedagogía consideran las experiencias prácticas como 
el componente más importante de su programa de formación inicial (Boyle-Baise \& Sleeter, 2000; Bullough et al., 2002; Clift \& Brady 2005).

Los docentes de aula señalan que el aprendizaje de la docencia se realiza en y por medio del ejercicio profesional y, en general, consideran las prácticas como los únicos momentos pertinentes de su formación. De igual forma, la práctica profesional docente contribuye a confrontar la representación de la profesión con la realidad del ejercicio profesional, la posibilidad de integrar los saberes provenientes de la teoría con los de práctica, la necesaria socialización profesional, la actualización de las competencias y el desarrollo profesional progresivo. Asimismo, el carácter profesionalizador de las prácticas permite poner en evidencia la responsabilidad individual y colectiva del desarrollo profesional (Correa, 2011). Estas Las anteriores son características reconocidas en la bibliografía que evidencian la importancia que ocupa la práctica pedagógica o debiera ocupar en los programas de formación docente.

Distintos autores coinciden que la reflexión acerca de la práctica pedagógica permite a sus actores considerar distintas perspectivas, logrando, sobre la base de ello, crear significados y conceptualizar su quehacer (Brockbank \& McGill, 1999; Fraile, 2000). De esta manera los futuros docentes parten de su propia experiencia y logran entrar en un proceso de crítica constructiva. Huerta (2011) agrega que la práctica favorece los procesos de cambio y renovación de la práctica docente, tanto del profesor como del colectivo en general.

Más concretamente, Fraile (2000) identifica tres ventajas que se desprenden de la reflexión acerca de la práctica docente. La primera de ellas, es la creación de instancias para enseñar contenido, pues la reflexión permite abordar desde otra perspectiva el actuar cotidiano en todas las actividades docentes y abre posibilidades de aprendizajes insertos en los mismos procesos que se están examinando. La segunda, dice relación con el descubrimiento progresivo que el docente hace realiza sobre su quehacer y la visión del aprendizaje que de ahí se deriva. Esto favorece un redescubrimiento de la cotidianidad profesional y también permite al profesor tomar decisiones conscientes sobre su actuar y por último la tercera ventaja, está relacionada con los fundamentos epistemológicos que subyacen en la práctica profesional docente. Es durante el desarrollo de la práctica profesional en la que los enfoques de intervención adquieren una visión crítica, generada por el contraste entre el conocimiento de las teorías y enfoques pedagógicos con el actuar práctico.

Schön (1998) explica cómo la reflexión sobre la práctica es un proceso construido a partir de una serie de vivencias profesionales que de forma progresiva transforman el pensar y hacer del sujeto que participa. Mientras que Por su parte Jay y Johnson (2002) entienden la reflexión de la práctica pedagógica como un proceso individual y colaborativo, que implica experiencia e incertidumbre, comprendiendo la identificación de cuestiones y elementos claves de una temática que es significativa, y cuyo proceso, permite lograr nuevas claridades, que permiten la modificación de la acción. De forma más reciente, Labra (2014) indica que la reflexión respecto de la práctica es una relación dialéctica teoría práctica que permite pensar de manera reflexiva los procesos de enseñanza aprendizaje.

Se desprende de los párrafos anteriores, la reflexión puede tener una variada gama de significados, dependiendo de la forma en la que se utilice en el campo del desarrollo profesional docente. La literatura describe numerosas fases, niveles, etapas, tipos o dimensiones de la reflexión, con caracterizaciones que van desde la simple consideración de un aspecto sencillo de una lección hasta las implicaciones éticas, sociales y políticas de la práctica pedagógica.

Las diversas definiciones, que han evolucionado por varias décadas, reflejan comúnmente tres niveles de reflexión. Así, Jay y Johnson (2002) propone los siguientes niveles: (i) descriptivo, que se focaliza en las estrategias y métodos de enseñanza utilizados para alcanzar objetivos educativos predeterminados; los docentes se preocupan más por lo que funciona en el salón de clases para mantener a los estudiantes en orden que por cualquier consideración en cuanto al valor 
que tienen tales objetivos y fines en sí mismos; (ii) comparativo, orientado a reflexionar sobre los objetivos educacionales, las teorías que subyacen a los diferentes enfoques de enseñanza y las conexiones que existen entre los principios teóricos y la práctica; (iii) crítico, que tiene como foco la reflexión acerca de las consecuencias de las prácticas educativas sobre los estudiantes, junto con la relación con las implicaciones éticas y morales de sus prácticas. Los docentes con reflexiones del tercer nivel son aquellos que extienden sus consideraciones hasta problemas más allá del salón de clases para incluir ideales democráticos. Ellos son los docentes involucrados en la reflexión pedagógica que buscan comprender la base teórica de la práctica en el aula (Villalobos, 2009), lo cual les permitiría realizar una extrapolación al ámbito donde se está realizando el acto educativo.

Además, para entender la manera en la que los profesores en formación van aprendiendo y desarrollando su propia identidad como docentes, es necesario adoptar metodologías prácticas que se centren en explicar las causas y la influencia aspectos tales como el actuar del profesor y estudiantes, actividades de aprendizaje y su evaluación, entre otros, como lo sugiere Pedder (2011). Sin embargo, lograr una reflexión desde los programas de práctica profesional es un desafío, pues en las universidades existe escasez de instancias y procesos que permitan desarrollar reflexión sistemática desde las prácticas profesionales (Labra, 2011; Nocetti de la Barra, Mendoza, Contreras, Sanhueza y Herrera, 2005). Por lo tanto, no es de extrañar que estas reflexiones se originen brote de actitudes individuales y no de un trabajo colectivo, guiado por las interrogantes: sobre qué, cómo y con qué fin enseñar, como lo advierte Labra (2011). En este sentido, los estudios de este autor indican que el nivel de reflexión de la práctica alcanzada por estudiantes de pedagogía es básico, es decir, los estudiantes en práctica emplean estrategias basadas en un conocimiento técnico y en las reacciones de sus propios estudiantes, existiendo descripción de situaciones y acciones. Ahora bien, los estudiantes de la carrera de Pedagogía Media en Ciencias Naturales y Biología de la Universidad Católica de Temuco presentan dificultad para realizar reflexión sobre su práctica pedagógica a partir de argumentos epistemológicos y empíricos que permitan generar cambios significativos en esta.

La problemática da cuenta de la necesidad de incorporar estrategias que permitan fortalecer procesos reflexivos de los estudiantes de pedagogía. Por lo tanto, son ellos quienes tienen el rol protagónico en el análisis de su práctica dado que son los interlocutores de su acción (Correa, 2011). De este modo, cuando el protagonista de un video analiza su propia acción propicia una comprensión de su actuar, discursos o actitudes.

De acuerdo con lo planteado por Rich y Hannafin (2009) el video ha sido utilizado para capturar episodios de microenseñanza, ilustrar casos y prácticas en el aula y revisar las prácticas docentes. Por lo tanto, el video hace posible la documentación, a la vez que apoya la reflexión sobre el trabajo de aula, facilitando examinar los cambios y el desarrollo de habilidades a través del tiempo. En esta misma línea, Altet (2000) posiciona a las grabaciones en video como detonadores del "recuerdo estimulado" o de la explicitación de las prácticas realizadas, de los saberes, del saber hacer y saber ser utilizados. Este material fílmico permite registrar la trayectoria docente y la habilidad de asociar el video capturado con la evidencia de desarrollo tanto del profesor como de los estudiantes. Son ambas características las que lo hacen idóneo para fomentar tanto el análisis como la reflexión del docente.

Al indagar sobre el efecto de la observación de videos en la reflexión y el desarrollo de habilidades docentes, Rosaen, Lundeberg, Cooper, Fritzen y Terpstra (2008), encontraron que el hecho de analizar videos de su trabajo posibilita a los profesores en formación hacer una reflexión más profunda sobre el manejo que hacen del aula y no sólo del comportamiento de los niños.

Como lo plantean Jay y Johnson (2002) la reflexión sobre la práctica pedagógica supone espacios de construcción individual, así como también colaborativos. Es entonces, que el trabajo en colegialidad cobra relevancia en el fortalecimiento de los procesos reflexivos de estudiantes de pedagogía. En este caso puntual, colegialidad se refiere a la participación de actores tales como 
profesores supervisores y estudiantes de pedagogía tutoreados por el mismo profesor supervisor. Correa (2011) expresa que "reflexionar en comunidad sobre su propia práctica y la de otro par puede, en cierta medida, tener un efecto positivo en la toma de consciencia sobre su propio actuar y de ese modo modificar la práctica sin que esto sea la intención explícita del investigador" (p.86). De esta manera, la problemática señalada se abordó a través de la implementación de una propuesta didáctica que incorporó la filmación de clase y su observación en contextos de colegialidad como estrategias para fortalecer procesos reflexivos sobre la práctica pedagógica en estudiantes de pedagogía.

Existen variadas acepciones para referirse a propuestas didácticas, Salinas (1990) la define como una forma existente para construir y organizar el conocimiento, que surge a partir de la identificación de un problema, ante el cual se plantean estrategias que permitan abordarlo. En una propuesta didáctica se deben considerar coherentemente todos los elementos que intervienen en el proceso de enseñanza aprendizaje, así como también el tiempo de desarrollo (Antúnez, 1992). Es así que objetivos, contenidos, actividades de aprendizaje y evaluación junto a elementos que contextualizan el proceso (nivel de desarrollo del alumno, medio sociocultural y familiar, proyecto curricular, recursos disponibles) organizan conocimientos y experiencias de enseñanza aprendizaje (Coll,1991).

La propuesta se articuló en base a un enfoque constructivista del aprendizaje (Ortiz, 2015) que permitió identificar conocimientos previos y construir nuevos significados realizando proceso de asimilación y acomodación de la información, acá los estudiantes adquirieron un rol activo y el docente fue una guía.

El objetivo de la propuesta fue fortalecer los niveles de reflexión de estudiantes de pedagogía. La estrategia que la guía es la observación, análisis y reflexión de filmaciones de clases en contextos de colegialidad, participando estudiantes en práctica pedagógica profesional y la profesora supervisora de esta práctica.

Los recursos que consideró la propuesta fueron: cuatro fichas de trabajo creadas para orientar a los participantes de la investigación a: (i) autoevaluar el nivel de reflexión inicial a partir de su desempeño en práctica profesional; (ii) filmar clases considerando aspectos metodológicos y éticos y (iii) observar clases considerando colegialidad, situación que permite identificar en el desempeño pedagógico de aula fortalezas, desafíos y orientar el proceso reflexivo de los participantes.

Para efectos de evaluación la estrategia utilizada, es el portafolio que permitió dimensionar los aprendizajes construidos y niveles de reflexión alcanzados por los participantes. La profesora supervisora es quien implementó la propuesta, guiando y monitorendo el proceso de autoevaluación, filmación y observación de clases y de igual forma, retroalimentó la reflexión sobre la práctica pedagógica.

\section{Método}

La investigación es de tipo cualitativo, está orientada a analizar casos concretos en su particularidad temporal y local y a partir de expresiones y actividades de las personas en sus contextos locales (Flick, 2004). El diseño corresponde a un estudio de caso único, considerado por Stake (2005) como aquel que permite la comprensión de la realidad objeto de estudio. El nivel de estudio es descriptivo. Los participantes fueron seleccionados intencionalmente y corresponden a dos estudiantes de práctica profesional de la carrera de Pedagogía Media en Ciencias Naturales y Biología de la Universidad Católica de Temuco.

Se emplearon las siguientes técnicas de recolección de datos: 
Análisis documental. De acuerdo con lo planteado por Peña y Pirela (2007) "es entendido como un proceso ideado por el individuo como medio para organizar y representar el conocimiento registrado en los documentos" (p. 59). Esta técnica facilitó la aproximación cognitiva del investigador al contenido de la fuente de información "autoevaluación" y "portafolio".

La autoevaluación, es entendida por Cabero y Gisbert (2002), como un conjunto de actividades autoreflexivas acompañadas de soluciones que permiten identificar el tipo y grado de aprendizaje respecto de los objetivos de la acción formativa. Esta, fue elaborada por los participantes a partir de la revisión de la primera entrega de portafolio que realizaron. Esta fuente de información identificó el nivel inicial de reflexión percibido por los participantes.

El portafolio "se constituye como una herramienta de mejora del aprendizaje basado en el pensamiento crítico sobre hechos de la práctica diaria, para favorecer la adquisición de conocimientos y habilidades de manera reflexiva, autodirigida y personalizada" (Universidad Católica de Temuco, 2017, p.50), el portafolio fue elaborado por los participantes. Se estructuró de acuerdo a lo expresado por el Ministerio de Educación (2001) en los Estándares de Desempeño para la Formación Inicial de Docentes, expresado en las facetas: A "Preparación de la enseñanza"; B "Ambiente para el aprendizaje"; C "Enseñanza para todos los estudiantes" y D "Profesionalismo Docente". Cada una de los participantes fue reflexionando respecto de su desempeño pedagógico en contraste con lo estipulado en los criterios e indicadores de las facetas antes señaladas. Para efectos de evaluación, este portafolio es entregado en cuatro momentos durante un semestre académico. No obstante, la información recopilada de los portafolios, fue el nivel de reflexión al inicio y término de la investigación, es decir, se trabaja con la primera y última entrega. Para este análisis se utilizaron como referencia los indicadores para los niveles de reflexión de Jay \& Johnson: descriptivo, comparativo y crítico.

Entrevista semiestructurada. Según Flick (2004), este tipo de entrevista reconstruye la teoría subjetiva del entrevistado sobre el problema de estudio. El instrumento Incorporó diez 10 preguntas orientadas a identificar percepciones de los participantes respecto de la incorporación del uso de filmaciones de clase en contexto de colegialidad para fortalecer el proceso de reflexión sobre la práctica y el nivel de reflexión que alcanzan.

El proceso de validación de la propuesta pedagógica y entrevista semiestructurada fue a través de juicio de expertos. Para el registro y análisis de datos, se obtuvo consentimiento informado de los participantes y se respetó la confidencialidad de identidad.

La triangulación metodológica permitió obtener información en diversas fuentes, formas y momentos. El análisis de los resultados de la investigación se realizó con el software ATLAS ti7.5, utilizando el proceso de codificación abierta, descrita como "el proceso analítico por medio del cual se identifican los conceptos y se descubren en los datos sus propiedades y dimensiones" (Flick, 2004, p. 193). Se procedió a la lectura de datos realizando codificación y categorización de estos.

Las categorías de análisis fueron:

(i) Percepción propuesta pedagógica: entendida como la apreciación de los participantes respecto de la elaboración e implementación de la propuesta que incorpora la estrategia de uso y análisis de las filmaciones de clase para el fortalecimiento de la reflexión sobre la práctica pedagógica.

(ii) Nivel de reflexión: entendido como el tipo de reflexión que alcanzan los participantes. Considerando lo planteado por Jay y Jonhson (2002), se categorizaron en descriptivo, comparativo y crítico. Este nivel de reflexión se analizó al iniciar y finalizar la investigación. 


\section{Resultados}

Los resultados de la investigación son presentados a partir de las siguientes categorías de análisis: Percepciones propuesta didáctica y Niveles de reflexión.

\section{Categoría Percepciones propuesta didáctica}

Las percepciones de los participantes fueron agrupadas en dos subcategorías: (i) percepción elaboración de propuesta didáctica y (ii) percepción implementación de propuesta didáctica.

\section{Subcategoría percepción elaboración de propuesta didáctica}

Al analizar la información, las percepciones de los participantes respecto de la elaboración de la propuesta didáctica se relacionan a los códigos redacción, procedimiento estructurado y proyecciones.

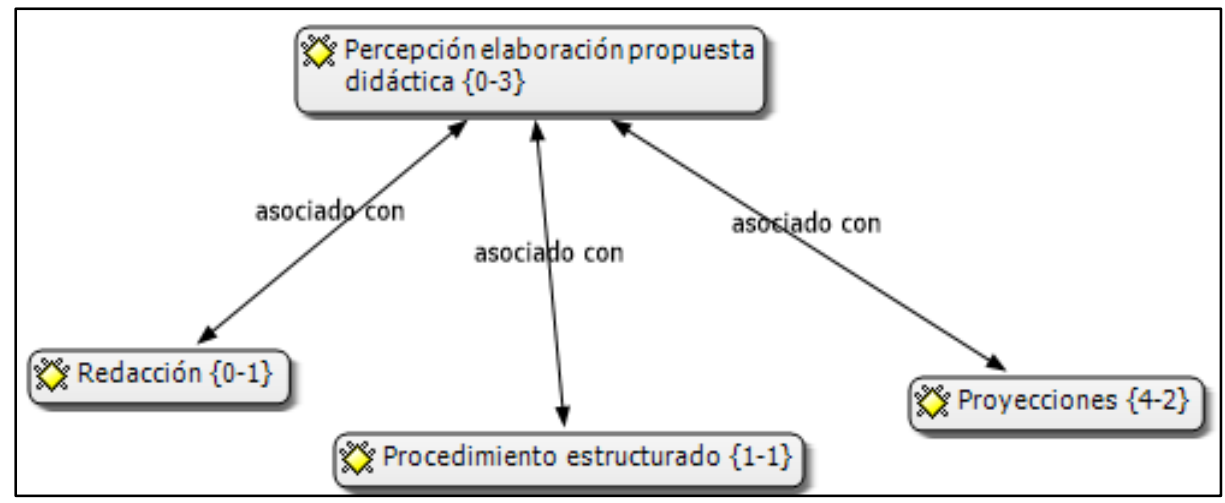

Figura 1. Percepción elaboración propuesta didáctica

En relación con el código redacción, los participantes indican la necesidad de revisar la claridad de las instrucciones, de forma de hacer mucho más comprensible la tarea a realizar. Atender a esta sugerencia es relevante, según Cassany (1990), la redacción es de gran importancia para que el contenido sea interpretado de modo inequívoco. De este modo, al realizar los cambios sugeridos por los participantes existirá una interpretación de lo solicitado con seguridad to que repercutirá de forma directa en los logros alcanzados por los participantes. Cabe destacar, que la profesora supervisora durante la implementación de la propuesta didáctica fue clarificando dudas lo que permitió la fluidez en el desarrollo de las acciones a realizar por los participantes.

El código procedimiento estructurado da cuenta que la propuesta didáctica estaba organizada, tal como se advierte en las citas: P1: Primero uno se observa y nosotros vamos reflexionando, yo lo hice en el portafolio. Iba comentando y pensando en lo que me decían mis compañeras y profesora supervisora, si hago esto lo puedo mejorar y ahí uno se va autocriticando y autoevaluando". El contenido de la cita reafirma lo expresado por Rosaen, Lundeberg, Cooper, Fritzen y Terpstra (2008) quienes señalan que el proceso reflexivo se fortalece a partir de la observación de filmaciones de la práctica pedagógica.

Si bien existe esta opinión, se levanta el tercer código, proyecciones para la propuesta pedagógica P2: "Me hubiese gustado que en las reuniones de trabajo que teníamos nos hubiésemos sentado a trabajar en el portafolio, pero no lo hicimos así"; P2: "lo que siempre tuve débil fue el cierre de clase. Por lo que creo que el trabajo también se debe focalizar en esa etapa de la clase". Como se puede apreciar, existe la necesidad de un acompañamiento estructurado para la elaboración del portafolio y además abordar estrategias que fortalezcan los momentos de la clase de forma específica el cierre de estas. Por ende, es necesario incorporar en la propuesta didáctica fichas de trabajo que permitan guiar el proceso de elaboración del portafolio. 
Si bien, los participantes reconocen que la propuesta didáctica con uso de filmaciones en contexto de colegialidad les permitió estructurar sus reflexiones manifiestan su preocupación por los permisos desde los establecimientos educacionales para realizar filmaciones, P1: "Si, lo único malo ahí es que algunos establecimientos impiden la filmación de clases lo que jugaría en contra en algunos alumnos pero si es necesario grabar". García (2008), afirma la importancia del uso de filmaciones ya que contribuye a la representación y construcción de la realidad en la medida en que captan, transforman y reelaboran hechos, situaciones y personajes, convirtiéndolos en productos, ya sean del carácter documental e informativo o de la ficción. Por tanto, y pese a las posibles dificultades con los establecimientos educacionales, es vital la existencia de estas para la reflexión sobre la práctica pedagógica.

De acuerdo a lo planteado, los profesores supervisores deben socializar de forma detallada protocolos de filmaciones de clase y consentimientos informados en los centros de práctica para resguardar la confidencialidad e integridad de los participantes, hecho que, sin duda, corresponde al campo de la ética profesional.

\section{Subcategoría percepción implementación de propuesta didáctica}

La información analizada respecto de las percepciones de la implementación de la propuesta didáctica informa, por una parte, la existencia de los códigos: autoobservación, evidencia explícita y organización que se relacionan al código fortalecimiento de la reflexión y por otra parte, los códigos proyecciones y guía del supervisor. Este último vinculado a los códigos retroalimentación y estilo estructurado.

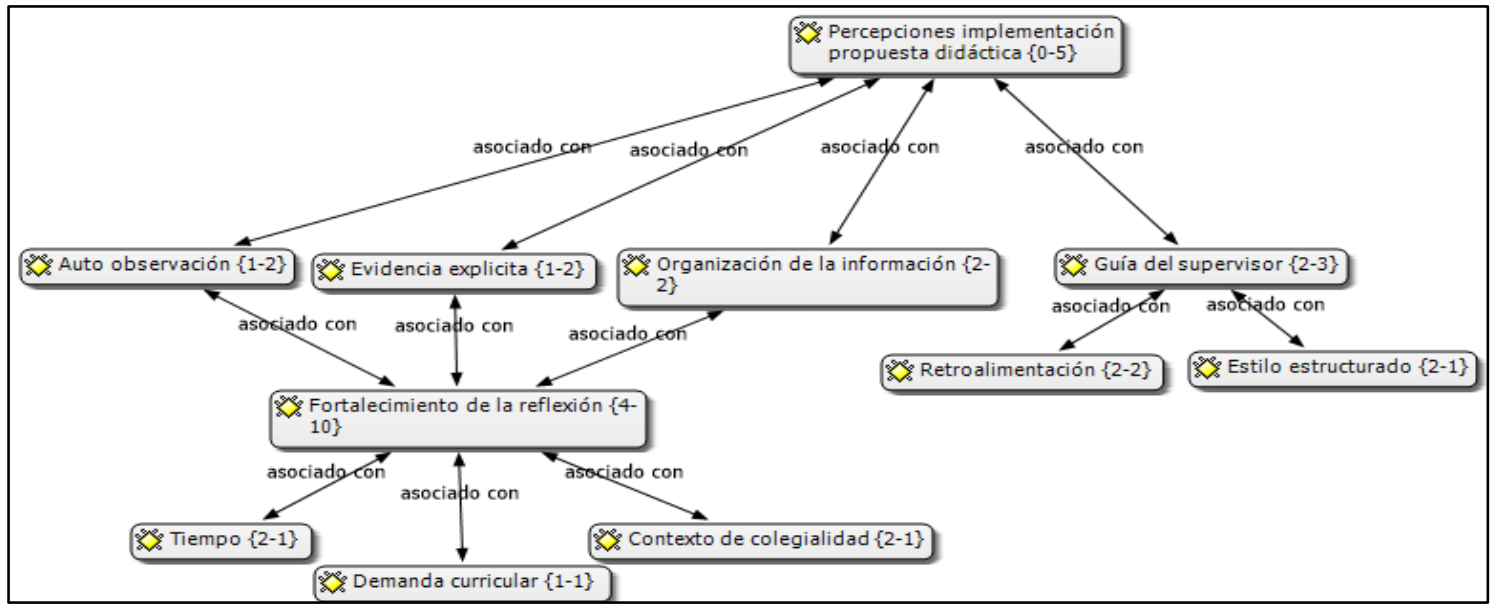

Figura 2. Percepción implementación propuesta didáctica

Para el código autoobservación la cita " $P 2$ El trabajo con videos me permitió observarme. Yo jamás había visto una clase mía por vergüenza, porque me daba risa, porque yo sabía que no me iba a gustar probablemente", informa que los participantes atribuyen que el uso de la filmación les permite observar su desempeño; sin embargo, se infiere la presencia de inseguridad en el actuar protagónico de aula. Por su parte el código evidencias explícitas respaldado en la siguiente cita "P1: si es necesario porque fortalece un proceso ya que uno hace una clase puede hacer el registro anecdótico pero el video respalda lo que efectivamente se hace en el aula" se constata la importancia del uso de filmaciones de clase pues representa la actuación del docente sin intervención de terceros.

En tanto el código organización de información, permite identificar dificultades para organizar la información, como se observa en el siguiente fragmento: "P2: Por ejemplo sé que en la parte de activación de conocimientos previos va en la faceta $C$ pero no supe donde agregarlo 
para que sirviera como pista. Pienso que me faltó reflexión, sentarme a analizar y a ver la pauta y criterios e indicadores de los Estándares de Formación Inicial docente”. Al respecto, Marcelo (2008) señala que los profesores noveles al no concretizar sus propias expectativas, además, de la carga docente y la falta de tiempo para reflexionar sobre su trabajo, dudan de su capacidad para resolver problemas y se sienten inseguros en su quehacer docente.

Las citas correspondientes a los códigos antes señalados advierten de un consenso en relación a la propuesta didáctica dado que esta fortaleció la reflexión sobre la práctica docente. Sin embargo, ambos participantes mencionan la dificultad de realizar reflexiones de alto nivel con los tiempos de trabajo personales y demandas curriculares de su plan de estudio con las que deben trabajar. "P1: tiempo influyo en la elaboración del portafolio y el tiempo que se requiere es esencial" "P2: los tiempos no me alcanzaban para las reflexiones profundas". Esto es consistente con lo manifestado por Zabalza (2007), quien comenta que los estudiantes, además, de aprender en situaciones concretas, poseen otras actividades que involucran tiempo y dedicación; es por ello que en muchas ocasiones no existe un compromiso real frente a tareas que se le asignan.

Los participantes señalan al trabajo en contexto de colegialidad como un factor importante en el fortalecimiento de la reflexión: "P1: A veces a uno no le gusta escuchar lo que opinan de uno pero es necesario lo que puede aportar los compañeros en el proceso de cada un"; "P2: el que mis otras compañeras me observaran y me dijeran esto es lo que tú no estás haciendo me indico que habían cosas que debía mejorar lo que se vio reflejado en las clases que me fueron a supervisar por ejemplo en cómo trabajar activación de conocimientos previos, cómo introducir nuevas estrategias pero lo que siempre tuve débil fue el cierre de clase”. Esto es respaldado por Correa (2011), puesto que el trabajo en contexto de colegialidad es una estrategia que fortalece procesos reflexivos con objetivo común, que es conocido y compartido por los estudiantes en formación de profesores y el profesor supervisor quien guía el proceso.

Existe reconocimiento de los participantes al profesor supervisor lo que se traduce en el levantamiento del código Guía del supervisor, quien promueve la retroalimentación "P1: se decía lo que se debía mejorar, pero el guiar no significó que me dieran la respuesta de cómo hacerlo, sino que yo me tenía que dar cuenta de cómo poder hacerlo. Me debía dar el trabajo de formular una estrategia para la mejora"; "P2: a lo mejor me hubieran dicho hazlo así, pero no, acá fue mira a lo mejor puedes hacer esto pero tú debes ingeniártelas, fue un proceso cooperativo". Montecinos (2011) afirma que la retroalimentación sistemática potencia la reflexión otorgando a los profesores en formación confianza en sus capacidades para asumir retos y complejidades propios al quehacer educativo.

A partir de las citas, se puede inferir que los participantes relacionan al profesor supervisor con un estilo estructurado " $P 1$ : Como nos guiaron a nosotros fue de manera estructura y eso hace que uno se guie de mejor manera. No al lote"; "Pl: La guía fue focalizada en los puntos que se debía fortalecer e ir preocupándose por los logros alcanzados para que no se perdieran"; "P2: Que el profesor supervisor guie aún más el proceso". Al respecto Ugartetxea (2002) afirma que incentivar al profesor en formación para que se haga cargo de su propio aprendizaje es fundamental para que tome conciencia de sus capacidades y competencias pedagógicas y, con ello, aumente sus creencias de autoeficacia.

\section{Categoría Nivel de reflexión}

Los resultados para la categoría nivel de reflexión, por una parte, da cuenta del nivel de reflexión que los participantes son capaces de autoevaluar en la primera entrega de su portafolio y, por otra parte, informa el nivel de reflexión inicial y final que la investigadora identifica en las entregas del portafolio. Como se mencionó anteriormente, el análisis se realiza a partir de los niveles de reflexión de Jay y Johnson (2002). 
Tabla 1.

Niveles de reflexión de participantes de la investigación identificaos en autoevaluación y entrega inicial y final de portafolio.

\begin{tabular}{|c|c|c|c|c|c|c|c|}
\hline \multirow{2}{*}{ } & \multirow{2}{*}{ } & \multicolumn{3}{|c|}{$\begin{array}{c}\text { Participante } 1 \\
\text { Nivel de reflexión }\end{array}$} & \multicolumn{3}{|c|}{$\begin{array}{c}\text { Participante 2 } \\
\text { Nivel de reflexión }\end{array}$} \\
\hline & & $\begin{array}{l}\text { Autoevalua } \\
\text { ción }\end{array}$ & $\begin{array}{c}\text { Entrega } \\
\text { inicial }\end{array}$ & $\begin{array}{c}\text { Entrega } \\
\text { final }\end{array}$ & $\begin{array}{l}\text { Autoevalua } \\
\text { ción }\end{array}$ & $\begin{array}{c}\text { Entrega } \\
\text { inicial }\end{array}$ & $\begin{array}{l}\text { Entrega } \\
\text { final }\end{array}$ \\
\hline \multirow{5}{*}{ A } & A1 & Descriptivo & Descriptivo & Crítico & Comparativo & Crítico & Crítico \\
\hline & A 2 & Descriptivo & Descriptivo & Crítico & Descriptivo & Comparativo & Comparativo \\
\hline & A3 & Descriptivo & Descriptivo & Crítico & Comparativo & Crítico & Crítico \\
\hline & A 4 & Descriptivo & Descriptivo & Crítico & Comparativo & Crítico & Crítico \\
\hline & A5 & Descriptivo & Descriptivo & Crítico & Comparativo & Comparativo & Comparativo \\
\hline \multirow[t]{5}{*}{ B } & B1 & Descriptivo & Descriptivo & Crítico & Comparativo & Comparativo & Crítico \\
\hline & B2 & Descriptivo & Descriptivo & Crítico & Comparativo & Comparativo & Crítico \\
\hline & B3 & Descriptivo & Descriptivo & Crítico & Comparativo & Comparativo & Crítico \\
\hline & B4 & Descriptivo & Descriptivo & Crítico & Comparativo & Comparativo & Crítico \\
\hline & B5 & Descriptivo & $\begin{array}{c}\text { Comparativ } \\
\mathrm{O}\end{array}$ & Crítico & Descriptivo & Comparativo & Crítico \\
\hline \multirow[t]{5}{*}{ C } & $\mathrm{C} 1$ & Descriptivo & Descriptivo & Crítico & Descriptivo & Comparativo & Comparativo \\
\hline & $\mathrm{C} 2$ & Descriptivo & Descriptivo & Crítico & Descriptivo & Comparativo & Comparativo \\
\hline & $\mathrm{C} 3$ & Descriptivo & $\begin{array}{c}\text { Comparativ } \\
\mathrm{o}\end{array}$ & Crítico & Descriptivo & Comparativo & Crítico \\
\hline & $\mathrm{C} 4$ & Descriptivo & Descriptivo & Crítico & Descriptivo & Comparativo & Crítico \\
\hline & $\mathrm{C} 5$ & Descriptivo & Descriptivo & Crítico & Descriptivo & Descriptivo & Comparativo \\
\hline \multirow{5}{*}{ D } & D1 & Descriptivo & Descriptivo & Comparativo & Sin reflexión & Descriptivo & Comparativo \\
\hline & D2 & Descriptivo & Descriptivo & Comparativo & Sin reflexión & Sin reflexión & Sin reflexión \\
\hline & D3 & $\begin{array}{l}\text { Sin } \\
\text { reflexión }\end{array}$ & $\begin{array}{l}\text { Sin } \\
\text { reflexión }\end{array}$ & Comparativo & Sin reflexión & Sin reflexión & Sin reflexión \\
\hline & D4 & $\begin{array}{l}\text { Sin } \\
\text { reflexión }\end{array}$ & $\begin{array}{l}\text { Sin } \\
\text { reflexión }\end{array}$ & Crítico & Sin reflexión & Sin reflexión & Sin reflexión \\
\hline & D5 & $\begin{array}{l}\text { Sin } \\
\text { reflexión }\end{array}$ & $\begin{array}{l}\text { Sin } \\
\text { reflexión }\end{array}$ & Comparativo & Sin reflexión & Sin reflexión & Sin reflexión \\
\hline
\end{tabular}

Los resultados expuestos en la tabla 1, informan que el participante un en la autoevaluación categoriza su reflexión en todas las facetas en el nivel descriptivo. Cabe destacar que la faceta $\mathrm{D}$ del portafolio solo presenta reflexión en dos criterios. En cuanto al nivel de reflexión alcanzado en la entrega inicial, esta participante presenta en todos los criterios de la faceta A nivel descriptivo. Lo mismo ocurre para los criterios de las facetas B, C y D. No obstante, en los criterios B5 y C3 se evidencia un nivel más bien comparativo. Para el caso de la entrega final, el nivel de reflexión alcanzado en las facetas A, B y C es crítico. En la faceta D la reflexión existente en los criterios D1, D2, D3 y D5 es de nivel comparativo y en el criterio D4 es crítico. El participante 2 en la autoevaluación evalúa en la faceta A su nivel de reflexión mayoritariamente comparativo, solo en el criterio A2 indica tener un nivel descriptivo. En la faceta B informa alcanzar el nivel de reflexión comparativo a excepción del criterio B5 que es descriptivo. En la 
Faceta C, para todos los criterios evidencia el nivel de reflexión descriptivo. La faceta D no presenta reflexión. En relación al nivel de reflexión alcanzado en la entrega inicial, esta participante presenta en los criterios A1, A3 y A4 de la faceta A nivel crítico. En los criterios A2 y A5 se observa el nivel comparativo. En los cinco criterios de la faceta B existe presencia del nivel reflexivo comparativo. Misma situación se presenta en los criterios C1, C2, C3 y C4; sin embargo, en el criterio C5 se identifica el nivel descriptivo. La faceta D, en el criterio D1 da cuenta del nivel de reflexión descriptivo. Criterios D2, D3, D4 y D5 no presentan reflexión. En la entrega final, el nivel de reflexión en la faceta A no presenta movilización, es decir se mantiene en los niveles informados en la entrega inicial. En la faceta B, en todos los criterios el nivel de reflexión alcanzado es el crítico. El nivel alcanzado en la faceta $\mathrm{C}$, criterios $\mathrm{C} 1, \mathrm{C} 2$ y $\mathrm{C} 5$ es comparativo; los criterios C3 y C4 dan cuenta de un nivel crítico. En la faceta $\mathrm{D}$ al igual que en la entrega inicial, el criterio D1 da cuenta del nivel de reflexión descriptivo. Criterios D2, D3, D4 y D5 no presentan reflexión.

\section{Discusión}

De acuerdo con los resultados de la aplicación de la propuesta didáctica con enfoque constructivista que incorporó la estrategia de filmación de clase en contexto de colegialidad permitió la estructuración del proceso de reflexión en relación a la práctica. De acuerdo a lo planteado por Rodrigues, (2013) la reflexión en la práctica supone un proceso cognitivo activo y deliberativo que necesariamente debe ser organizado para adoptar una postura más crítica del desempeño docente. Según Richards y Lockhart (2007) al reflexionar críticamente el profesor se siente más confiado en incorporar diferentes opciones didácticas y evaluar el impacto de estas. En esta misma línea, Correa, Chaubet, Collin y Gervais (2014) argumentan que "la presencia de un "otro", que interpela y tranquiliza a la vez, contribuiría a la explicitación de la acción y a la reflexión sobre la acción" (p.79).

El uso de la filmación como lo afirman, Rich y Hannafin (2009), Altet (2000) y Rosaen, Lundeberg, Cooper, Fritzen y Terpstra (2008), permite extraer evidencias explícitas de prácticas de aula que estimulan el recuerdo y con ello el proceso reflexivo de los saberes que sustentan el desempeño docente. Por tanto, es necesario que esta acción de autoobservación sea instalada y fortalecida en el proceso formativo de los estudiantes en formación de profesor.

El trabajo colegiado entre profesor supervisor y estudiantes en práctica fue muy-bien valorado dado que el profesor supervisor acompañó, guió y retroalimentó el proceso reflexivo. En otras palabras, fomentó la reflexión acerca de la práctica pedagógica, permitiendo una movilización de los niveles de reflexión. Larrivee (2008) señala que la reflexión no es un proceso lineal, sino que un sujeto podría estar en diferentes niveles de manera simultánea. Sin embargo, es perentorio incorporar el acompañamiento guiado del profesor supervisor cuando se esté elaborando el portafolio en las sesiones de trabajo colegiado. De esa manera se incentivaría a los estudiantes a dedicar tiempos específicos para realizar esta acción. Para dicho efecto cobra relevancia, la estrategia de argumentación práctica promovida Fenstermacher (1996), puesto que fomenta la identificación y verbalización de premisas de acción que fundamentan el actuar docente, entre estas podemos encontrar conocimientos teóricos, saber de experiencia, valores, elementos contextuales.

Para posteriores implementaciones de la propuesta didáctica, la redacción debe ser clara, lo que permitirá el desarrollo de actividades en el menor tiempo posible, redestinando parte de este a la elaboración del portafolio en contexto de colegialidad, situación que da mayor seguridad a los participantes al momento de analizar su desempeño considerando aspectos epistemológicos. La información recabada en esta investigación, da cuenta de la necesidad de continuar con el proceso, proyectándolo a otras cohortes de la carrera de Pedagogía Media en Ciencias Naturales y Biología. Asimismo, se considera importante poder realizar un estudio del estilo de profesores supervisores que acompañan a los estudiantes de Internado Pedagógico de esta misma carrera, dado que son los participantes de este estudio que cursaron práctica profesional quienes valoraron 
el rol del profesor supervisor como guía que retroalimenta y acompaña procesos desde un estilo estructurado, pero no directivo, que logra dar espacios para reflexionar al estudiante.

\section{Referencias}

Antúnez, S. (1992). Del Proyecto Educativo a la Programación de Aula. El qué, el cuándo y el cómo de los instrumentos de la planificación didáctica. Barcelona: Graó.

Altet, M. (2000). Les dispositifs d'analyse des pratiques pédagogiques en formation d'enseignants: une démarche d'articulation pratique-théoriquepratique", en: Claudine Blanchard-Laville y Dominique Fablet, L'analyse des pratiques professionnelles, Paris, L'Harmattan.

Boyle-Baise, L., \& Sleeter, C. E. (2000). Community-based service learning for multicultural teacher education. Educational Foundations, 14(2), 33-50.

Bullough, R. V., Young, J., Erickson, L., Birrell, J.R., Clark, D. D., Egan., M. W., Berrie, F., Hales, V., \& Smith, G. (2002). Rethinking Field Experience: Partnership teaching versus single -placement. Teaching, 3, 68-80.

Brockbank, A., \& McGill, I. (1999). Aprendizaje reflexivo en la educación superior. Madrid, España: Morata.

Cabero, J., \& Gisbert, M. (2002). La evaluación, Materiales formativos multimedia en la red. Guía práctica para su diseño. Sevilla: SAV

Cassany, D. (1990). Enfoques didácticos para la enseñanza de la expresión escrita. Comunicación, lenguaje y educación, 6(2). doi: https://doi.org/10.1080/02147033.1990.10820934

Coll, C. (1991). Psicología y currículum. Barcelona. Paidos.

Coll, C., Martín, E., Mauri, T., Miras, M., Onrubia, J., Solé, I., \& Zabala, A. (1999). El constructivismo en el aula. Barcelona: Graó.

Correa, E. (2011). La práctica docente: una oportunidad de desarrollo profesional. Perspectiva Educacional, 50(2), 77-95.

Correa, E., Chaubet, P.,Collins, S., \& Gervais,C. (2014) Desafíos metodológicos para el estudio de la reflexión en contexto de formación docente. Estudios Pedagógicos, XL (Número Especial 1), 71-86

Clift, R. T., \& Brady, P. (2005). Research on methods courses and field experiences. En M. Cochran-Smith, y K. M. Zeichner (eds.) Studying Teacher Education: The report of the AERA Panel on Research and Teacher Education (pp. 309-424). Mahwah, NJ: Lawrence Erlbaum Associates.

Fenstermacher, G. (1996). Les arguments pratiques dans la transformation morale del'enseignement d'une discipline. Revue des sciences de l'éducation, 22(3), 617-634

Flick, U. (2004). Introducción a la Investigación Cualitativa. España: Ediciones Morata S.L.

Fraile Aranda, A. (2000). Factores obstaculizadores y facilitadores en la formación del profesorado de educación física. En Contreras Jordán, O. R. La formación inicial y permanente del profesor de educación física. Castilla La Mancha: Ediciones de la Universidad de Castilla-La Mancha.

García, M. (2008). El vídeo como herramienta de investigación. Una propuesta metodológica para la formación de profesionales en Comunicación. Facultad de Comunicación Social para la Paz Universidad Santo Tomás. Bogotá, Colombia.

Huerta, P. (2011) XI Congreso Nacional de Investigación Educativa Monterrey Nuevo León México.

Jay, J. \& K. Johnson. (2002). Capturing complexity: a typology of reflective practice for teacher education. Teaching and Teacher Education. 18, 73-85

Labra, P. (2011). Construcción de conocimiento profesional docente: el caso de la formación en la práctica (Tesis doctoral) Universidad Academia de Humanismo Cristiano, Santiago de Chile.

Larrivee, B. (2008). Development of a tool to assess teachers' level of reflective practice. Reflective Practice, 9(3), 341-360. https://doi.org/10.1080/14623940802207451 
Marcelo, C. (2008). El profesorado principiante: Inserción a la docencia. Barcelona, España: Octaedro.

Ministerio de Educación de Chile (2001). Estándares de Desempeño para la Formación Inicial de Docentes. Santiago, Chile.

Montecinos, C. (2011) Relación entre estilos de supervisión durante la práctica profesional y las creencias de autoeficacia de los estudiantes de Pedagogía en Educación Básica. Perspectiva Educacional, 50(2), 96-122.

Nocetti de la Barra, A., Mendoza, M., Contreras, G., Sanhueza, K., \& Herrera, S. (2005). Caracterización de las prácticas iniciales de las carreras de pedagogía en universidades chilenas. Concepción: Universidad San Sebastián - FONIDE.

Ortiz,G. (20015). El constructivismo como teoría y método de enseñanza. Sophia, Colección de Filosofía de la Educación, 19, 93-110.

Pedder, V. D. (2011). Conceptualizing teacher profesional learning. Review of Educational Research 81(3), 376 - 407. doi: https://doi.org/10.3102/0034654311413609

Peña, T., \& Pirela, J. (2007). La complejidad del análisis documental. Información, cultura y sociedad: Revista del Instituo de Investigaciones Bibliotecológicas, 16, 55-81.

Rich, P., \& Hannafin, M. (2009). Video Annotation Tools: Technologies to Scaffold, Structure, and Transform Teacher Reflection. Review of Journal of Teacher Education 60(52). doi: https://doi.org/10.1177/0022487108328486

Richards, J. C., \& Lockhart, C. (2007). Reflective Teaching in Second Language Classrooms. New York: Cambridge University Press.

Rodrigues, R. (2013). El desarrollo de la práctica reflexiva sobre el quehacer docente, apoyada en el uso de un portafolio digital, en el marco de un programa de formación para académicos de la Universidad Centroamericana de Nicaragua (Tesis de Doctorado). Recuperado de http://hdl.handle.net/2445/43124

Rosaen, C., Lundeberg, M., Cooper, M., Fritzen, A. \& Terpstra, M. (2008). Noticing, noticing. How does investigation of video records change how teachers reflecto $n$ their experiences? Journal of Teacher Education 59(4), 347-360. doi: https://doi.org/10.1177/0022487108322128

Salinas, G. (1990). La Propuesta pedagógica en las licenciaturas de educación preescolar y educación primaria en lengua indígena. Recuperado de https://es.scribd.com/doc/104463555/1-Gisela-Salinas-La-Prop-Ped

Schön, D. (1998). El profesional reflexivo: cómo piensan los profesionales cuando actúan. Barcelona: Paidós Ibérica.

Stake, R. E. (2005). Investigación con estudio de casos. Madrid: Morata.

Universidad Católica de Temuco (2017). Marco Orientador de la Formación Práctica.

Ugartexea, J. (2002). Metacognición, el desarrollo de la autoeficacia y la motivación. Revista Psicodidactica, 13, 49-77.

Villalobos, J., \& M de Cabrera, C. (2009). Los docentes y su necesidad de ejercer una práctica reflexiva. Revista de Teoría y Didáctica de las Ciencias Sociales,14(1), 139-166.

Zabalza, M. (2007). La enseñanza universitaria. El escenario y sus protagonistas. Madrid: Narcea. 